\title{
Fusimotor control of spindle sensitivity regulates central and peripheral coding of joint angles
}

\author{
Ning Lan ${ }^{1,2 *}$ and Xin $\mathrm{He}^{1}$ \\ 'School of Biomedical Engineering, Med-X Research Institute, Shanghai Jiao Tong University, Shanghai, China \\ ${ }^{2}$ School of Dentistry, Division of Biokinesiology and Physical Therapy, University of Southern California, Los Angeles, CA, USA
}

\section{Edited by:}

Hava T. Siegelmann, University of

Massachusetts Amherst, USA

Reviewed by:

Meng Hu, Drexel University, USA Dimitri Nowicki, Moscow Institute of

Physics and Technology, Ukraine

\section{*Correspondence:}

Ning Lan, School of Biomedical

Engineering, Med-X Research

Institute, Shanghai Jiao Tong

University, 1954 Hua Shan Road,

Shanghai 200030, China.

e-mail: ninglan@sjtu.edu.cn
Proprioceptive afferents from muscle spindles encode information about peripheral joint movements for the central nervous system (CNS). The sensitivity of muscle spindle is nonlinearly dependent on the activation of gamma $(\gamma)$ motoneurons in the spinal cord that receives inputs from the motor cortex. How fusimotor control of spindle sensitivity affects proprioceptive coding of joint position is not clear. Furthermore, what information is carried in the fusimotor signal from the motor cortex to the muscle spindle is largely unknown. In this study, we addressed the issue of communication between the central and peripheral sensorimotor systems using a computational approach based on the virtual arm (VA) model. In simulation experiments within the operational range of joint movements, the gamma static commands $\left(\gamma_{\mathrm{s}}\right)$ to the spindles of both mono-articular and bi-articular muscles were hypothesized (1) to remain constant, (2) to be modulated with joint angles linearly, and (3) to be modulated with joint angles nonlinearly. Simulation results revealed a nonlinear landscape of la afferent with respect to both $\gamma_{s}$ activation and joint angle. Among the three hypotheses, the constant and linear strategies did not yield la responses that matched the experimental data, and therefore, were rejected as plausible strategies of spindle sensitivity control. However, if $\gamma_{s}$ commands were quadratically modulated with joint angles, a robust linear relation between la afferents and joint angles could be obtained in both mono-articular and bi-articular muscles. With the quadratic strategy of spindle sensitivity control, $\gamma_{s}$ commands may serve as the CNS outputs that inform the periphery of central coding of joint angles. The results suggest that the information of joint angles may be communicated between the CNS and muscles via the descending $\gamma_{\mathrm{s}}$ efferent and la afferent signals.

Keywords: muscle spindle, $\gamma_{\mathrm{s}}$ control, spindle sensitivity, la afferents, joint angle, central and peripheral coding

\section{INTRODUCTION}

Muscle spindle is a unique sensory organ that has dual efferent and afferent innervations (Boyd, 1980; Matthews, 1981; Hulliger, 1984). A large amount of cortical outputs is directed to $\gamma$ motoneurons that supply fusimotor control of spindles (Boyd and Smith, 1984). A larger number of studies have been dedicated to elucidate the morphological, biochemical, and neurophysiological properties of the spindle (Matthews, 1962; Granit, 1970; Boyd and Smith, 1984). But relatively little has been revealed about the functional role of fusimotor efferent in the execution of motor tasks, because it has been difficult, if not impossible, to record directly from gamma motor neurons during normal movements. Fusimotor control is so far best understood to adjust the sensitivity of muscle spindles. As the alpha motor neurons activate extrafusal muscle fibers to produce a contraction force, the spindle is unloaded. To keep the spindle sensitive during muscle contraction, the central nervous system (CNS) may coactivate the intrafusal fiber via descending gamma commands $\gamma$ (Vallbo and al-Falahe, 1990), in order to assess the outcome of the alpha activation of muscles. In so doing, if $\gamma_{\mathrm{s}}$ command were properly modulated with movement, the spindle firing may not be interrupted by the unloading effects of muscle contraction. Early studies have associated the spindle function to regulation of muscle length (Merton, 1953; Stein, 1974; Houk and Rymer, 1981). But difficulties of the length-servo hypothesis have turned the direction of research towards more centrally organized programming for motor control (Flash and Hogan, 1985; Feldman, 1986; Hasan, 1986; Corcos et al., 1989; Gottlieb et al., 1989; Uno et al., 1989; Harris and Wolpert, 1998; Todorov and Jordan, 2002). On the other hand, central programming or coding of sensorimotor control must take into account the peripheral constraints presented in the neuromuscular system (Kawato et al., 1990; Lan and Crago, 1994; Lan, 1997). Thus, it is necessary to elucidate the nature of information communicated between the central and peripheral systems.

It has been a main subject of experimental studies with regard to the nature of gamma fusimotor commands relevant to motor control (Boyd, 1980; Matthews, 1981; Hulliger, 1984; Boyd et al., 1985). Only until recently, experimental studies of patterns of gamma motor activity during movement and posture in animals have shed some light to the plausible function of fusimotor co-activation with $\alpha$ commands (Taylor et al., 2004). 
Direct recordings from gamma fibers in reduced cat preparations showed that there was in-phase modulation of $\gamma_{s}$ activities with muscle EMGs during locomotion, providing firm evidence of $\alpha-$ $\gamma$ co-activation during movement (Taylor et al., 2000). And static gamma activity was considered to be a fusimotor template of intended movement (Taylor et al., 2006). This implied that $\gamma_{s}$ signal might carry centrally planned kinematic information of joint angles. In the periphery, direct recording of $I a$ afferents from the dorsal ganglion cells of decerebrated cats indicated a robust linear relation between $\mathrm{I} a$ afferents and joint angles (Stein et al., 2004). In human subjects, direct recording of spindle afferents from the extensor carpi radialis brevis (ECRb) and extensor digitorum (ED) (Cordo et al., 2002) revealed that the steady-state population firing of $I a$ afferents was found linearly related to joint position during the hold period between ramps. In these experiments, $\gamma_{s}$ modulation of spindle sensitivity was unknown in both animal and human recordings. However, the evidence in reduced animal preparations and intact human subjects provided partial clues on the central and peripheral coding of joint positions by fusimotor $\left(\gamma_{\mathrm{s}}\right)$ commands and Ia afferent signals.

In a more theoretical approach, a number of studies have suggested that trajectory and final position of movement may be planned separately, and executed with a dual control strategy (Lan et al., 2005; Ghez et al., 2007; Scheidt and Ghez, 2007). Experimental evidence also indicated that the brain treats movement and position information with distinct neural representations (Kurtzer et al., 2005). Injection of the $\gamma$-aminobutyric acid (GABA) antagonist picrotoxin into cat's reticular part of the substantia nigra (SNR) removed static fusimotor action from spindle primary endings (Wand and Schwarz, 1985). On the other hand, electrical stimulation at fasciculus retroflexus region of the cat's midbrain reproduced dynamic fusimotor effect, indicating that the habenulo-interpeduncular system may be involved in generating dynamic gamma commands (Taylor and Donga, 1989). Thus, movement and position control signals may be generated and processed in different regions of the brain, and passed down to spinal motor neurons as separate descending commands (Lemon, 2008). A set of static commands may be most relevant to maintaining a steady state limb position (Lan et al., 2005), while a set of dynamic commands may control dynamic acceleration and deceleration of movements (Lan and Crago, 1994; Lan, 1997; Lan et al., 2005). In this framework of dual control, it is necessary that the CNS inform the peripheral neuromuscular system about the desired joint position via a pathway separate from the $\alpha$ commands to the muscles. Recent experimental data (Cordo et al., 2002; Taylor et al., 2006) imply that an alternative pathway for transmission of kinematic information is via $\gamma$ commands to muscle spindles.

In this study, we used a computational approach to explore the functional role of fusimotor system in transmitting the centrally planned joint kinematics to the periphery, and how a robust linear relation between $\mathrm{I} a$ afferent and joint angle could be achieved with fusimotor control of spindle sensitivity. With a computational model of the virtual arm (VA) (Song et al., 2008a; He et al., 2012), we tested three plausible strategies of fusimotor control of spindle sensitivity with constant, linear and nonlinear modulations with joint angles. The correlation between joint angles and $I a$ afferents under different fusimotor control strategies were investigated for mono-articular and bi-articular muscles. The hypotheses were rejected or accepted based on the consistence of simulated behaviors to those of experiments. Part of the preliminary results was presented in a conference proceeding (He and Lan, 2011).

\section{MATERIALS AND METHODS THE SENSORIMOTOR SYSTEMS MODEL}

The computational model of the integrated, multi-joint sensorimotor VA system used in this study was shown in Figure 1. This model has been developed and validated for simulation studies of neural control of human arm movements (Song et al., 2008a; He et al., 2012). The VA model was capable of generating Ia afferents of muscles at different joint angles and under different fusimotor inputs. Thus, it was suitable to address the issue of how fusimotor control affects the coding of joint angles by Ia afferents. For completeness, a succinct description of the systems model was given below.

The VA systems model in Figure 1 was a two-joint arm in the horizontal plane. It consisted of subcomponent models of an anatomically accurate upper arm with shoulder and elbow joints, and physiologically realistic muscles and proprioceptors. Each model component has been validated respectively during its development (Cheng et al., 2000; Mileusnic et al., 2006; Song et al., 2008a,b), and then integrated into the realistic VA systems model in SIMULINK (Figure 1).

Computational modules of the VA model were implemented with a graphic modeling software SIMM and SIMULINK, respectively. The mathematical equations of geometry, kinematic, and dynamics of the multi-body system of the upper arm were embodied into SIMM, and the SIMM model was converted into a computational block in SIMULINK that computed joint motion with given muscular forces acting upon the joints (Song et al., 2008a). There were six representative muscles acting on the joints. The virtual muscle (VM) model contained all mathematical equations that described realistic muscle physiology and mechanics (Cheng et al., 2000), and a new version of the VM model was implemented in SIMULINK (Song et al., 2008b). The VM module computed muscle force and muscle fascicle length with given neural input after a continuous recruitment scheme (Song et al., 2008b). The new VM model improved computational efficiency and simulation stability. It allowed a continuous recruitment of slow and fast fibers, and decoupled $\alpha, \gamma$ command inputs to active extrafusal and intrafusal fibers, respectively.

Three pairs of agonist and antagonist muscles were selected to actuate two degrees of freedom (DOF) of the VA model in horizontal plane (Figure 1). Pectoralis major (clavicle portion, PC) and Deltoid posterior (DP) were mono-articular flexor and extensor at the shoulder joint; brachialis (BS) and triceps brachii lateral head (Tlt) were mono-articular flexor and extensor of the elbow joint; biceps brachii short head (Bsh) and triceps brachii long head (Tlh) were the bi-articular muscles cross both joints.

Each muscle model was embedded with a spindle model (Mileusnic et al., 2006) and a simplified Galgi tendon organ (GTO) model (Song et al., 2008a). The spindle model contained a bag1, a bag2, and a chain fiber with $I a$ and $I I$ afferent outputs. 


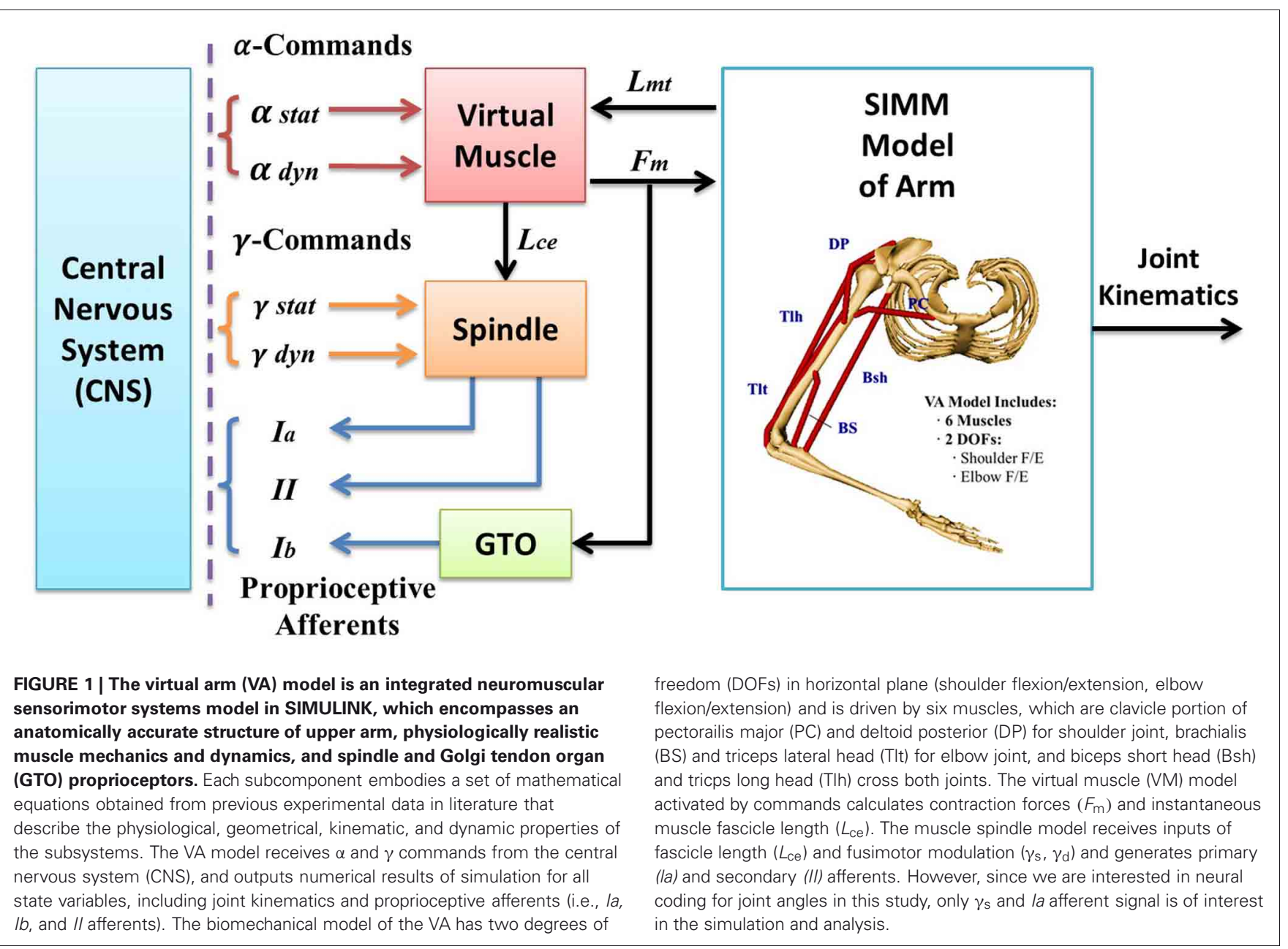

I $a$ afferents were sum of all fiber outputs and II afferents were primarily from chain fibers. The gamma static efferent innervated bag2 and chain fibers, and the gamma dynamic efferent innervated primarily bagl fiber. Thus, the spindle model was capable of simulating spindle $I a$ and $I I$ responses to both static and dynamic fusimotor inputs.

\section{DETERMINATION OF A SET OF EQUILIBRIUM POSITIONS IN SIMULATION}

The definitions of shoulder and elbow angles were shown in Figure 2A. The range of shoulder flexion was set from $0^{\circ}$ (fully extended) to $120^{\circ}$ (fully flexed), and the range of elbow flexion was from $0^{\circ}$ (fully extended) to $150^{\circ}$ (fully flexed). Showing in Figure 2A were a typical mono-articular muscle crossing the elbow joint, and a typical bi-articular muscle crossing both shoulder and elbow. The spindles were arranged in parallel with muscle fascicle fibers. In this study, however, joint angles of shoulder and elbow were varied in the operational range within the full range of motion (ROM), as shown in Figure 2B.

A procedure of initialization for dynamic simulation used in (He et al., 2012) was adopted in this study to obtain a set of equilibrium positions as shown in Figure 2B. The $\alpha$ commands of the nine stable equilibrium positions were tabulated in Table $\mathbf{1 .}$
The procedure was effective to determine initial system parameters, such as, fascicle length and joint angles, so that simulation could converge and the shoulder and elbow joints could be stabilized to a desired equilibrium position. In each simulation, the total running time was about 30 (s), in which the initial $10 \mathrm{~s}$ were designed to allow simulation to converge. A random, signal dependent noise (SDN) (Jones et al., 2002) was added to the muscle activation (He et al., 2012) at about 10 (s) to reproduce the inherent variability in the neuromuscular system. The steady state joint angles and $I a$ afferents were calculated as the average value of data in the last $10 \mathrm{~s}$ of simulation.

At the set of equilibrium positions, the geometric relationships between joint angle and muscle fascicle length in all muscles was evaluated. This was one of the peripheral constraints for the central programming of control of both intrafusal and extrafusal fibers. The joints of the VA were placed to different angles in the workspace (Figure 2B) by choosing particular patterns of mono-articular muscle activations (Table 1). The musculotendon lengths of the muscles were calculated from the VA model and the corresponding fascicle lengths were obtained at these joint angles. The relationship between joint angles and muscle fascicle length in the operational range of joint movement was then assessed in Figure 3. 


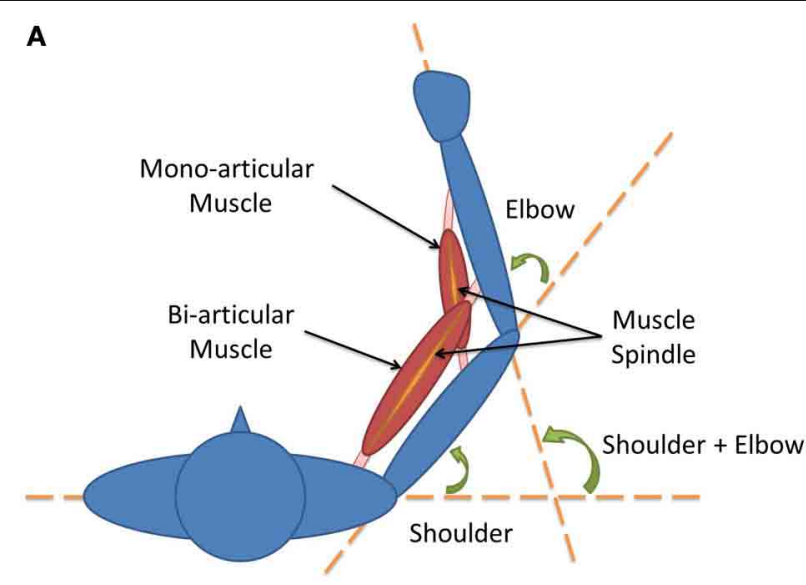

B

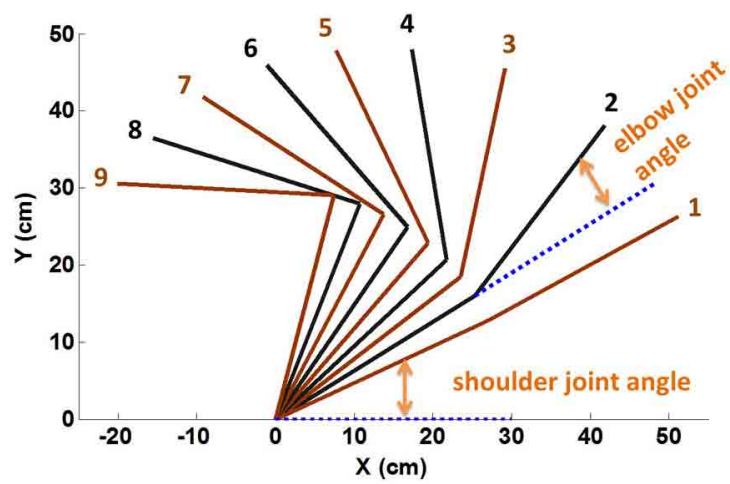

FIGURE 2 | (A) Geometric definition of shoulder and elbow angles. The range of shoulder flexion is set from $0^{\circ}$ (fully extended) to $120^{\circ}$ (fully flexed), and the range of elbow flexion is from $0^{\circ}$ (fully extended) to $150^{\circ}$ (fully flexed). Showing in the figure are a typical mono-articular muscle crossing the elbow joint, and a typical bi-articular muscle crossing both shoulder and elbow joints. The spindles are arranged in parallel with muscle fascicle fibers. We hypothesize that muscle fascicle length $\left(L_{c e}\right)$ and $l a$ afferent are related to the corresponding joint angles of span. Thus for bi-articular muscles, they are related to the sum of joint angles of span. (B) Nine sets of $\alpha_{\text {stat }}$ commands (Table 1) are used to stabilize the VA model at nine equilibrium positions $(1 \sim 9)$ in horizontal plane, respectively. At each position the spindle sensitivity control by $\gamma_{\text {stat }}$ is investigated.

\section{EVALUATION OF SPINDLE SENSITIVITY}

In this study, we focused on the effects of gamma static, $\gamma_{\mathrm{s}}$, control of spindle sensitivity with respect to muscle fascicle length change, while the gamma dynamic control was fixed to a constant level. An example of influences of gamma static control and joint angles on spindle sensitivity was explored in the six muscles. First, at a fixed joint configuration, the gamma static commands to all muscles were varied in a ramp pattern, and the Ia afferents of the six spindles showed simultaneous variation with the ramp change of the gamma static command. Then, the sensitivity of Ia afferent to fascicle length change was examined in response to ramp changes in joint angles with constant levels of gamma static inputs in the six muscles. These results were shown in Figure 4, and they verified the Ia sensitivity
Table 1 | Alpha static activation levels at nine positions.

\begin{tabular}{llllllllll}
\hline Muscle & \multicolumn{10}{c}{ Position } \\
\cline { 2 - 11 } & $\mathbf{1}$ & $\mathbf{2}$ & $\mathbf{3}$ & $\mathbf{4}$ & $\mathbf{5}$ & $\mathbf{6}$ & $\mathbf{7}$ & $\mathbf{8}$ & $\mathbf{9}$ \\
\hline PC & 0.25 & 0.30 & 0.35 & 0.40 & 0.45 & 0.50 & 0.55 & 0.60 & 0.65 \\
DP & 0.65 & 0.60 & 0.55 & 0.50 & 0.45 & 0.40 & 0.35 & 0.30 & 0.25 \\
Bsh & 0 & 0 & 0 & 0 & 0 & 0 & 0 & 0 & 0 \\
Tlh & 0 & 0 & 0 & 0 & 0 & 0 & 0 & 0 & 0 \\
BS & 0.25 & 0.30 & 0.35 & 0.40 & 0.45 & 0.50 & 0.55 & 0.60 & 0.65 \\
Tlt & 0.65 & 0.60 & 0.55 & 0.50 & 0.45 & 0.40 & 0.35 & 0.30 & 0.25
\end{tabular}

to both gamma static control and joint angle (or fascicle length).

The landscape of spindle Ia sensitivity with respect to joint angles and gamma static control was then further evaluated. In these simulations, alpha $\left(\alpha_{s}\right)$ commands represented the activation level of motor neuron pool as inputs to the VA model. Each set of constant motor commands $\left(\alpha_{s}, \gamma_{s}\right)$ produced an equilibrium position of the arm with $I a$ afferents from six muscles. A total of nine sets of alpha commands to the shoulder and elbow muscles (Table 1) positioned the VA model to nine different equilibrium angles in the shoulder and elbow joints (Figure 2B). The gamma static $\left(\gamma_{s}\right)$ commands were changed from 0.0 to 1.0 with an increment of 0.1 at each of the joint angles. A total of 81 points in the $\theta_{\mathrm{EP}}-\gamma_{\mathrm{s}}-I a$ space formed the landscape surface of Ia sensitivity for each muscle (Figure 5), which revealed the fundamental relationship among the three variables.

\section{TEST OF FUSIMOTOR CONTROL STRATEGIES}

Three sets of simulation experiments were designed to evaluate the plausible strategies regarding spindle sensitivity control. Based on the shape of the sensitivity landscape in the $\theta_{\mathrm{EP}}-$ $\gamma_{s}$ - Ia space, fusimotor control strategies, represented by the relation between equilibrium angle and gamma static command $\left(\gamma_{\mathrm{s}}-\theta_{\mathrm{EP}}\right)$, were hypothesized (1) to remain constant for all joint angles (H1); (2) to be modulated linearly with joint angles (H2); and (3) to be modulated quadratically with joint angles (H3). The resultant relation between Ia afferents and joint angles was evaluated under the three hypotheses of fusimotor control, and the outcome relation of the $\left(I a-\theta_{\mathrm{EP}}\right)$ curve was compared to experimentally observed behaviors. The necessary condition to reject a hypothesis was that the outcome relation of Ia afferents with joint angles must be a linear relation (Cordo et al., 2002; Stein et al., 2004). But to form a sufficient set of conditions to accept a hypothesis, other physiological constraints of experimental evidence in addition to the linear $\left(I a-\theta_{\mathrm{EP}}\right)$ output must be considered.

\section{RESULTS}

\section{THE LENGTH-ANGLE RELATION OF MUSCLES}

The relation between muscle fascicle length and joint angle revealed a geometric constraint in the VA model. The geometric relation was characterized by the $\theta_{\mathrm{EP}}-L_{\mathrm{ce}}$ curves shown in Figure 3 for the six muscles. In the operational range of shoulder and elbow joints, simulation results showed that the fascicle 


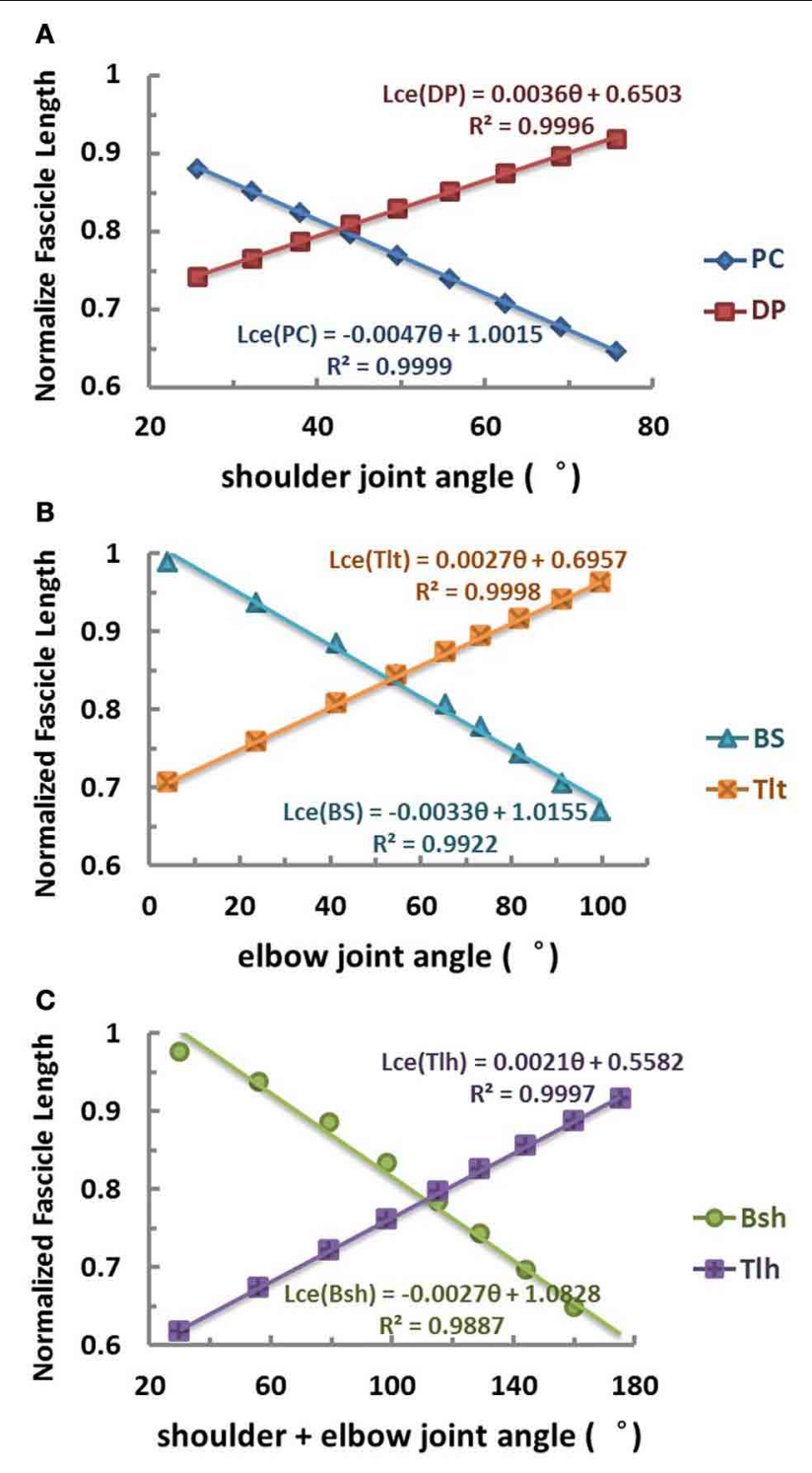

FIGURE 3 | Relation between equilibrium joint angle and muscle fascicle length $\left(\theta_{E P}-L_{c e}\right)$ of each muscle obtained in the range of joint angles used in simulation. (A) Relation of shoulder mono-articular muscles PC and DP. (B) Relation of elbow mono-articular muscles BS and TIt. (C) Relation of bi-articular muscles Bsh and Tlh cross both shoulder and elbow joints. Results indicate that a nearly linear relation exists between muscle fibre length and joint angle for both mono-articular muscles and bi-articular muscles, because of the their arrangement. The fascicle length of flexor is shortened and that of extensor is lengthened with increase of the joint angles of span. For bi-articular muscles Bsh and Th, their fascicle length, $L_{c e}$, is found linearly related to the sum of shoulder and elbow angles. The linearity in the geometric relations provides supportive evidence for a simple coding relationship between joint angles and spindle input and output that are related to muscle fascicle length

length of both mono-articular and bi-articular muscles was linearly related to the joint angles they cross. For mono-articular muscles, the joint of span was either shoulder joint or elbow joint, and thus the fascicle length of mono-articular muscles was linearly proportional to either shoulder angle or elbow angle
A
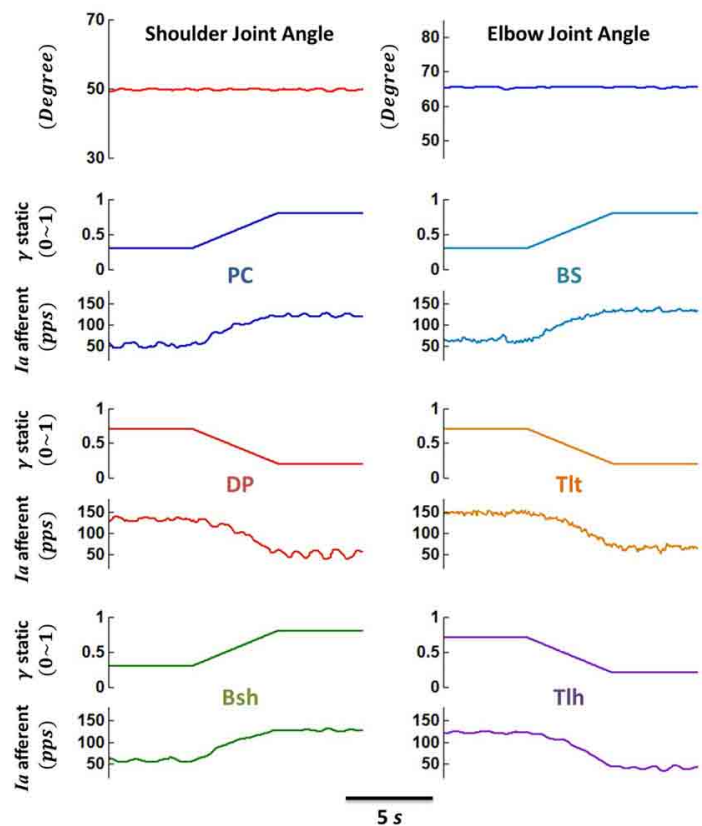

B
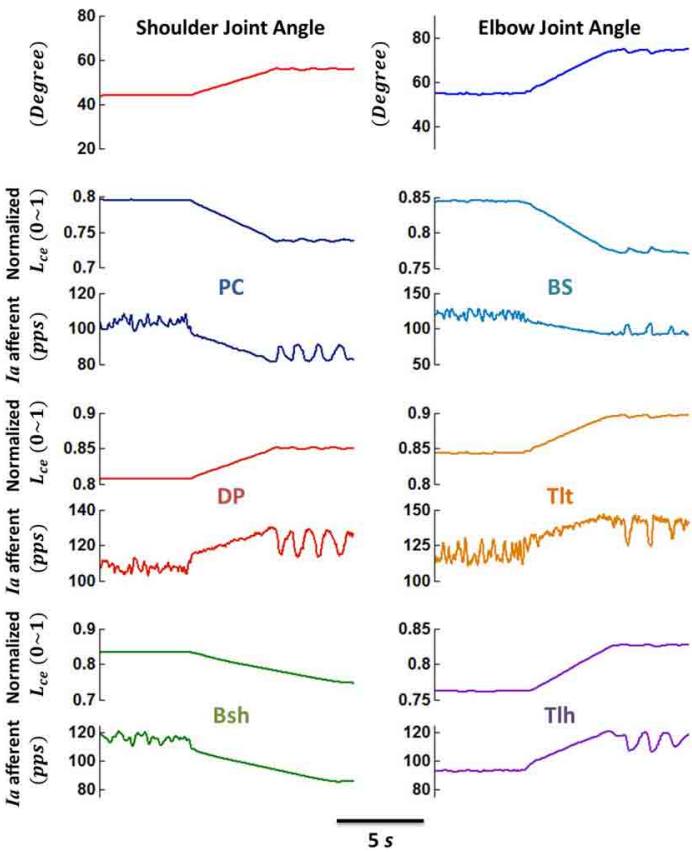

FIGURE 4 | Responses of primary afferents (la) of muscle spindles to (A) ramped $\gamma_{\mathrm{s}}$ drive, and (B) ramped joint angle (fascicle length) change, respectively. (A) The VA was maintained at position 5 , while $\gamma_{s}$ commands of flexors ramped from 0.3 up to 0.8 and those of extensors ramped from 0.7 down to 0.2 , concurrently within $5 \mathrm{~s}$. The la afferents of all muscles were shown to be modulated in-phase with $\gamma_{\mathrm{s}}$ changes. (B) The VA was moved from position 4 to 6 within $5 \mathrm{~s}$ by ramped alpha commands of single joint muscles, while the $\gamma_{s}$ commands of each muscle remained constant at 0.5 . The muscle fascicle length changed simultaneously with joint angles, and the la afferents were modulated in-phase with changes in fascicle length $L_{c e}$. These demonstrate the sensitivity la afferents with respect to fusimotor activation $\gamma_{s}$ and muscle fascicle length $L_{c e}$. 
(Figures 3A,B). For bi-articular muscles, the joints of span were both shoulder and elbow joints, and thus the fascicle length was linearly proportional to the sum of shoulder and elbow angles (Figure 3C). It is worth to note that while this fitted relation was near linear within the operational range of joints, significant nonlinearity may occur at the two extremes of ROM of joints, because the wrap around curvature of the joint was most effective within the operational ROM. Thus, at the extreme of joint angles, the CNS may rely on additional modality of proprioception, such as joint receptors, to estimate the value of joints accurately.

\section{SPINDLE RESPONSES TO CHANGES IN GAMMA STATIC AND FASCICLE LENGTH}

Figure 4 illustrated that gamma fusimotor control and fascicle length changes can modulate the sensitivity of Ia afferents effectively. The primary (Ia) afferents of muscle spindles responded to ramped gamma static drive (Figure 4A) and ramped fascicle length (Figure 4B) differently. In the simulations for fusimotor modulation effects, the VA was stabilized at position 5, and the gamma static commands of flexors were ramped from 0.3 up to 0.8 and those of extensors from 0.7 down to 0.2 during a period of $5 \mathrm{~s}$. It was shown that the $I a$ afferents of all muscles were modulated in proportion with gamma static changes (Figure 4A), showing a strong modulation of Ia sensitivity by gamma static commands. In the simulation for joint angle changes, the VA was moved from position 4 to 6 during a period of $5 \mathrm{~s}$. Joint angles were ramped from initial position to destination with linear changes in alpha drives to single joint muscles, while gamma static commands of each muscle remained constant at 0.5 . Figure $4 \mathrm{~B}$ showed the response of the Ia afferents to joint angle (or muscle fascicle length) changes. Clearly, there was a speed sensitivity component in the Ia response that was not present in isometric gamma static sensitivity of Figure 4A. After reaching to the destination of joint position, the spindle outputs were generally settled to a new level, but were affected by the dynamics in the spindle model (Mileusnic et al., 2006), as well as noise in the neuromuscular system (Jones et al., 2002). In this case, the fascicle length variation was about $10 \%$, and the $I a$ outputs at steady state were varied approximately with the similar percentage, indicating effective modulation of $I a$ sensitivity by joint angles (or fascicle length).

\section{THE LANDSCAPE OF SPINDLE SENSITIVITY}

In order to obtain a general view of spindle $I a$ afferents in the normal range of joint angles and with all possible gamma static values, we searched the joint angle-fusimotor commandprimary afferent $\left(\theta_{\mathrm{EP}}-\gamma_{\mathrm{s}}-I a\right)$ space. In these simulations, the joint angles were fixed to one of the nine positions in Figure 2B. Then the gamma static inputs to the six muscle spindles were varied from 0.1 to 1.0 , and $I a$ afferents of the six muscle spindles were examined. The results of each muscle were plotted in the 3D graphs in Figure 5. It was shown in general that the response of $I a$ afferents was not linear with respect to either joint angles or fusimotor commands in the whole space. This was not surprise because of the nonlinear response of spindles to both fusimotor commands and fascicle length change. Nonlinearity mainly occurred at the shorter muscle length, where the intrafusal fibres were relaxed at lower gamma activation levels. On the other hand, saturation of $I a$ afferents at longer muscle length with higher gamma activation levels also gave rise to nonlinear spindle sensitivity. The landscape of Ia sensitivity depicted the interrelation between gamma static commands and joint angles that may dictate the fusimotor control strategy.

\section{PLAUSIBLE FUSIMOTOR CONTROL STRATEGIES}

If joint angle is coded in the descending $\gamma_{\mathrm{s}}$ commands in the CNS, the $\gamma_{s}$ should be formulated as a function of joint angle, so that the resultant relation between $I a$ afferents and joint angles matches to that of experimentally observed linear relation. Thus, the central strategy of joint angle coding must take into account of the spindle sensitivity with respect to joint angles and fusimotor activation in Figure 5. Since the CNS has the luxury to control spindle sensitivity by fusimotor commands, the CNS may modulate spindle sensitivity by adjusting the central pattern of coding of joint angles, in order to keep the peripheral coding of joint angle by I $a$ afferents consistent and reliable under all conditions. The modulation of $\gamma_{s}$ command may occur in a relatively narrow region, for example a constant $\gamma_{s}$ value; or in a wide range of $\gamma_{s}$ command between 0.0 and 1.0 in order to achieve a better resolution of coding. In this study, we examined three scenarios (hypotheses) regarding fusimotor control strategy: (1) $\gamma_{s}$ was maintained constant within the range of joint angle; (2) $\gamma_{s}$ was modulated with joint angle in a linear function of joint angle; and (3) $\gamma_{s}$ was modulated in a nonlinear function with joint angles.

The first scenario was tested by postulating that $\gamma_{s}$ commands remained constant at modest activation levels within the entire range of joint angle $\theta$ (Figures 6A-C). The primary afferents of mono-articular muscle spindles showed a good linear relationship with corresponding joint angles (Figures 6D,E). The Ia afferents of bi-articular muscle spindles were also linearly proportional to the sum of shoulder and elbow joints as well (Figure 6F). The Ia afferents of extensor DP, Tlt, and Tlh were positively proportional to the joint angles, and Ia afferents of flexor PC, BS, and Bsh were negatively proportional to joint angles (Table 2). However, it was clear from Figure 5 that such good linear relations in $\theta_{\mathrm{EP}}-$ Ia were only possible from median to high levels of $\gamma_{s}$ activations. A constant fusimotor control may not be a physiological strategy because this does not allow any central coding of joint angles with $\gamma_{s}$. In addition, experimental evidence did not support constant $\gamma_{\mathrm{s}}$ commands during movements (Taylor et al., 2000, 2004, 2006).

The second scenario was then examined with $\gamma_{s}$ varied linearly with joint angle $\theta$ (Figures 7A-C). The linear relation of $\theta_{\mathrm{EP}}-\gamma_{\mathrm{s}}$ could be learned by the CNS to specify desired position of joints. However, the results of Ia afferents of both monoarticular and bi-articular muscle spindles were not linearly related to joint angles, as was shown in Figures 7D-F. This nonlinear response was evident from the nonlinear landscape of Ia sensitivity shown in Figure 5, where nonlinearity occurred in the lower and higher regions of gamma activation and joint angles. As a result, there was saturation in the Ia response as indicated by the arrows in Figure 7. Thus, the outcome of the linear hypothesis of fusimotor control strategy did not give rise to a linear relation 

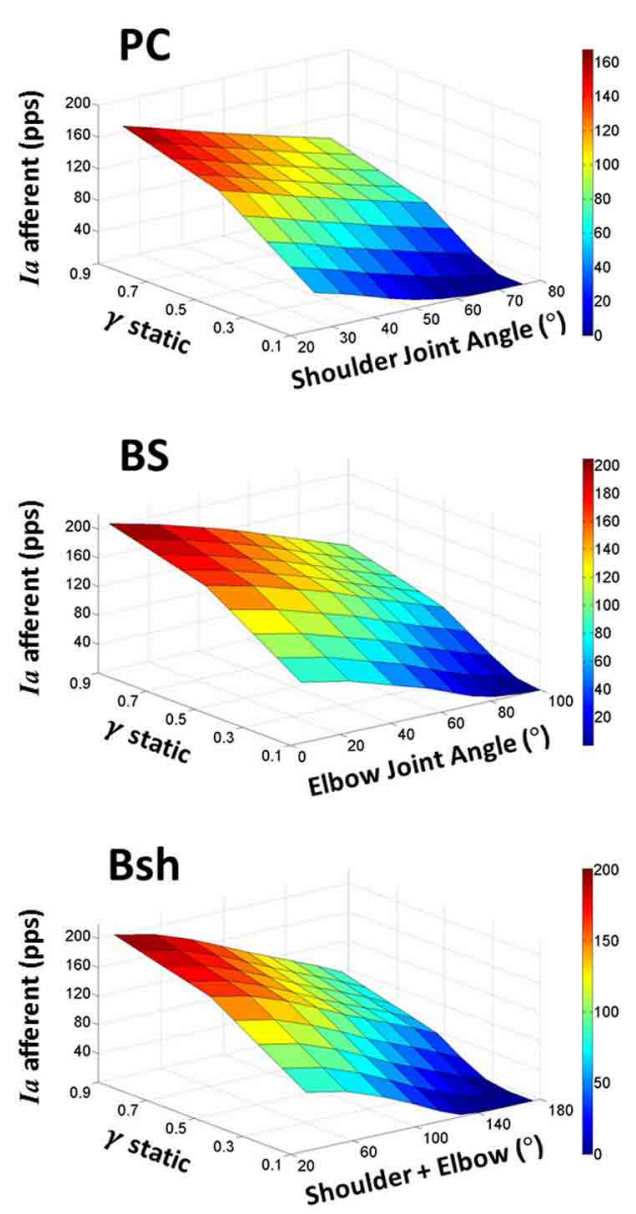

FIGURE 5 | The landscape of la afferent sensitivity with respect to the full ranges of fusimotor commands and muscle fascicle lengths

$\left(\theta_{\mathrm{EP}}-\gamma_{\mathbf{S}}-\mathbf{l a}\right)$. This is obtained by increasing fusimotor drive at each joint angle for all muscles incrementally. The sensitivity landscapes clearly reveal the complex interrelations of la afferents with both fusimotor control and joint angles. In general, the interrelation is nonlinear, and the nonlinearity is more
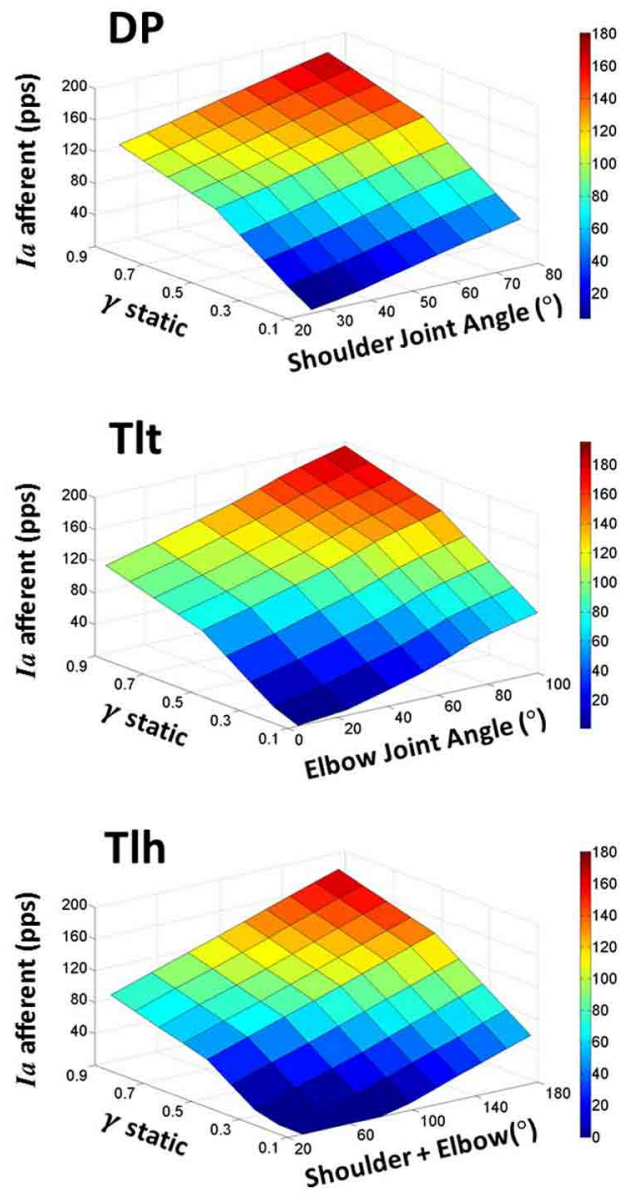

prominent at the lower and higher values of fusimotor commands and joint angles. These nonlinearities may be due to sluggish sensitivity in the short fascicle length and saturation in the long fascicle length. However, it is also clear that the nonlinearity exists in the middle range of fusimotor commands for all muscles. This phenomenon reflects the nonlinear nature of physiological responses of muscle spindles. in $\theta_{\mathrm{EP}}-$ Ia that was observed in experiments (Cordo et al., 2002; Stein et al., 2004). Therefore, the hypothesis of linear central coding of joint angle by $\gamma_{\mathrm{s}}$ command was rejected as a plausible strategy of fusimotor control.

Consequently, this led us to consider the third scenario of a nonlinear monotonic coding of joint angle by fusimotor command, which would yield a linear output in $I a$ afferents with respect to joint angles. We hypothesized that $\gamma_{s}$ commands were modulated quandratically with joint angle $\theta$ in parabolic curves of $\theta_{\mathrm{EP}}-\gamma_{\mathrm{s}}$, as shown in Figures 8A-C for the six muscles. Results showed that the Ia afferents of the six muscles were well correlated with joint angles linearly, as shown in Figures 8D-F, with a goodness of fitting $R^{2}>0.99$. The outcome of this strategy appeared to fit all experimental data available (Cordo et al., 2002; Stein et al., 2004), and thus it may be the most likely strategy that the CNS may adopt for fusimotor control of spindle sensitivity. Note that although the central coding relationship between $\theta_{\mathrm{EP}}-\gamma_{\mathrm{s}}$ is non-linear, it remains a monotonic curve. This implies that $\gamma_{s}$ could encode joint position uniquely within the ROM of joints. This phenomenon suggests that it is possible to manipulate the fusimotor commands to linearize the nonlinear Ia sensitivity revealed in Figure 5. The coefficients of fitted equations are presented in Table 3, which may be used in future simulation studies.

\section{DISCUSSION}

The understanding of neural control of movement would not be complete without revelation of the nature of fusimotor control for intrafusal fibers. The presence of enormous efferent and afferent innervations in the spindle prompts the question of what functional implications this sophisticated system may have, given that there is a vast CNS neural circuitry dedicated to process efferent and afferent neural information. The afferent signals may provide the CNS with peripheral kinematic information that allows the CNS to assess the outcome of executed motor action. But it is not straightforward as to what 


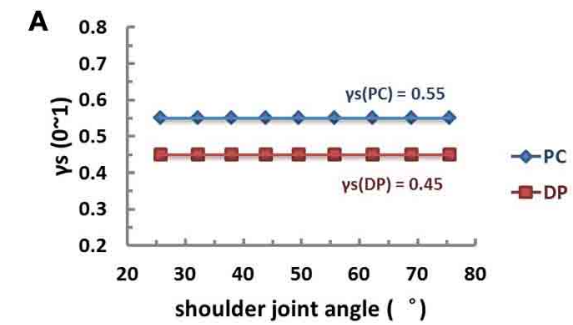

D

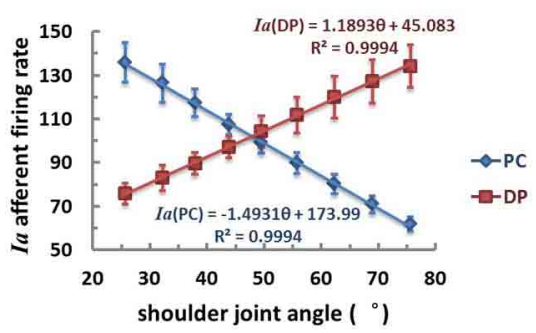

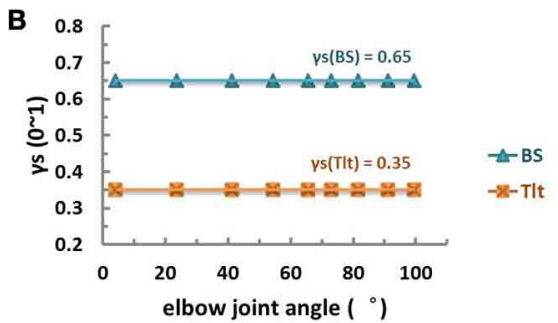

E

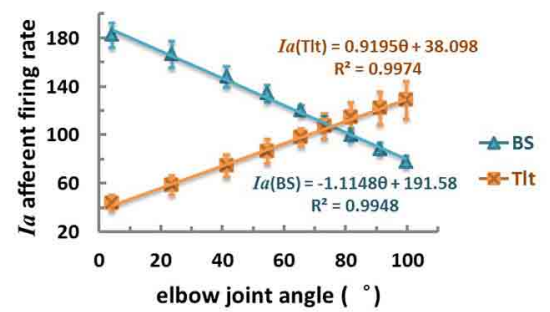

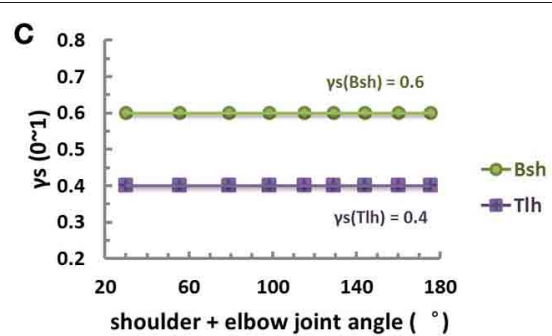

$\mathbf{F}$

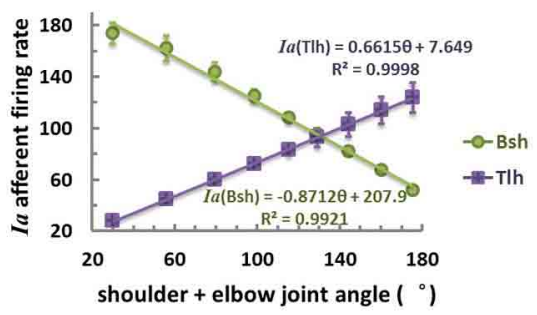

FIGURE 6 | The constant fusimotor control strategy, $\theta_{\mathrm{EP}}-\gamma_{\mathrm{S}}$, curves $(A-C)$, and $l a$ afferents response $\left(\theta_{\mathrm{EP}}-\mathrm{la}\right)$ curves of muscle spindles (D-F), for each muscle. The $(\mathbf{A}, \mathbf{D})$ is for shoulder actuators, the $(\mathbf{B}, \mathbf{E})$ is for elbow actuators, and the (C,F) is for bi-articular muscles. The abscissas indicate joint flexion angles while the vertical axis indicate activation levels (A-C) or firing rates (D-F). The $\gamma_{s}$ inputs of muscle spindles are set to medium constant levels at different angular positions, the firing rates of la afferents show an excellent linear relation with joint angles (with a goodness of fitting $R^{2}>0.99$ ). This result may be evident from the sensitivity landscape of Figure 5, from which a constant $\gamma_{S}$ value results in a fairly linear $\left(\theta_{E P}-\right.$ la) relation.
Table 2 | Fitting coefficients of constant $\gamma_{\mathrm{s}}$ strategy.

\begin{tabular}{|c|c|c|c|c|c|c|}
\hline & \multicolumn{3}{|c|}{$\gamma_{s}=a \theta^{2}+b \theta+c$} & \multicolumn{3}{|c|}{$\mathrm{la}=\mathbf{k} \theta+\mathrm{e}$} \\
\hline & $\mathbf{a}$ & b & c & $\mathbf{k}$ & e & $\mathbf{R}^{2}$ \\
\hline $\mathrm{PC}$ & 0 & 0 & 0.55 & -1.4931 & 173.99 & 0.9994 \\
\hline $\mathrm{DP}$ & 0 & 0 & 0.45 & 1.1893 & 45.083 & 0.9994 \\
\hline$B S$ & 0 & 0 & 0.65 & -1.1148 & 191.58 & 0.9948 \\
\hline Tlt & 0 & 0 & 0.35 & 0.9195 & 38.098 & 0.9974 \\
\hline Bsh & 0 & 0 & 0.60 & -0.8712 & 207.90 & 0.9921 \\
\hline TIh & 0 & 0 & 0.40 & 0.6615 & 7.6490 & 0.9998 \\
\hline Average & & - & & $\begin{array}{r}1.0416 \\
(p p\end{array}$ & 0.2644 & 0.9972 \\
\hline
\end{tabular}

information content is carried in the efferent fusimotor signals to the spindle in the periphery. A more compelling fact is that there are more neurons in the motor cortex that innervate spinal gamma motoneurons than those that control alpha motoneurons (Boyd and Smith, 1984). Thus, it is imperative to understand the significance of the large amount of corticospinal outflows of fusimotor control to the peripheral musculoskeletal system.

There have been early efforts to identify indirectly the profiles of fusimotor control signals during motor performance (Hulliger and Prochazka, 1983; Hulliger et al., 1987). This was largely conducted with Ia afferent data recorded from reduced preparations of passively behaving animal models. An inverse simulation method was proposed to deduce the fusimotor profile from recorded $I a$ afferents along with movements. However, it was then realized that the fusimotor profile could be entirely different in voluntarily behaving animals from those in reduced animal models. Also, there were intermediate variables, such muscle fascicle length, musculotendon length and joint angle, that may all affect the accuracy of estimates. A more stringent limit was due to the nonlinear dynamics of the musculoskeletal responses, which may lead to a non-unique pattern of fusimotor activity, even though optimization technique may help reduce the uncertainty of estimation.

In spite of technical difficulty, recently, direct recording from gamma motor neurons in reduced animal models had successfully produced considerable insight into the fusimotor activation profiles during movements (Taylor et al., 2000, 2004, 2006). It was observed that static gamma activity formed a fusimotor template of intended movement (Taylor et al., 2006), and may carry kinematic information of joint angles. In the periphery, direct recording of I a afferents from the dorsal ganglion cells of decerebrated cats suggested a similar conclusion that $I a$ afferents carried joint angle information (Stein et al., 2004). Similar conclusion was confirmed in human subjects with a voluntary contraction task (Cordo et al., 2002), which revealed that the steady-state population firing of Ia afferents was found linearly related to joint position during the hold period between ramps. Notice that in the experiments of Stein et al. (2004) and Cordo et al. (2002), the $\gamma_{s}$ modulation of spindle sensitivity was unknown. But separate recordings of fusimotor activities and $I a$ afferents suggested that fusimotor efferents may be programmed in the CNS such that Ia afferents reliably inform the kinematics of peripheral limb movements. More importantly, these experimental results formed a set of necessary conditions that simulated behaviors of muscle spindle apparatus must conform. In the sense of mathematical proof using computational methods, a 

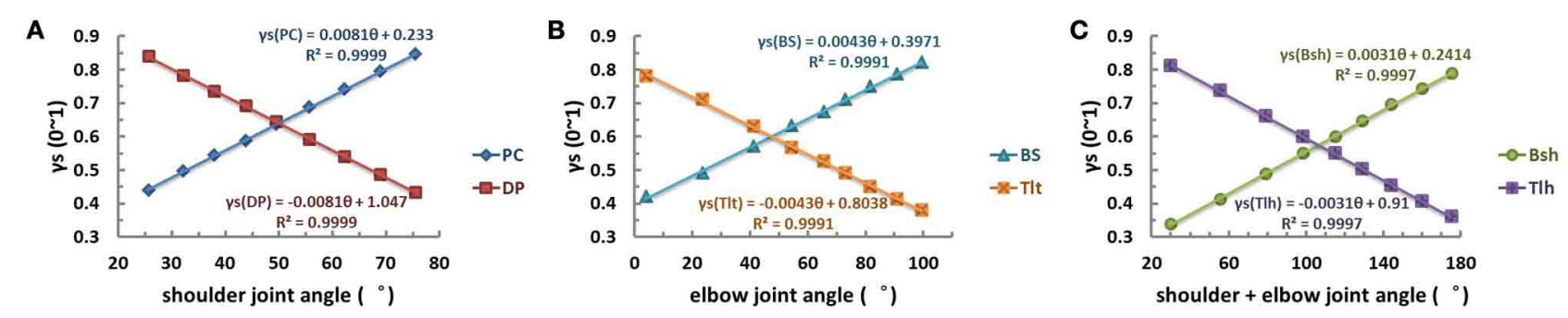

D

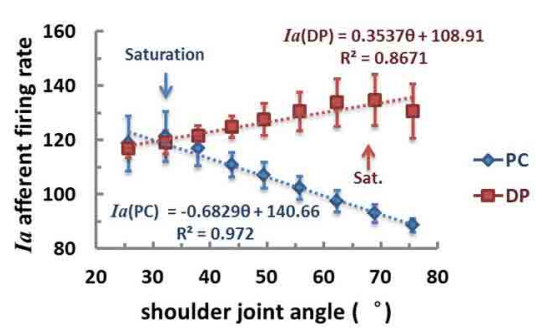

E

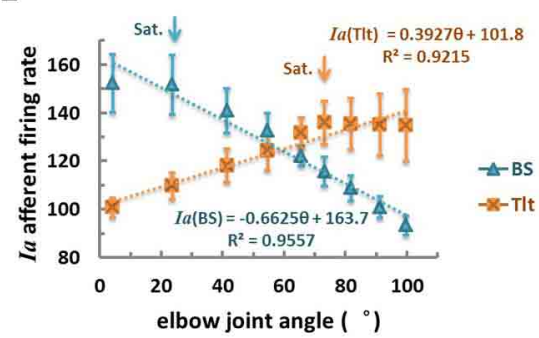

$\mathbf{F}$

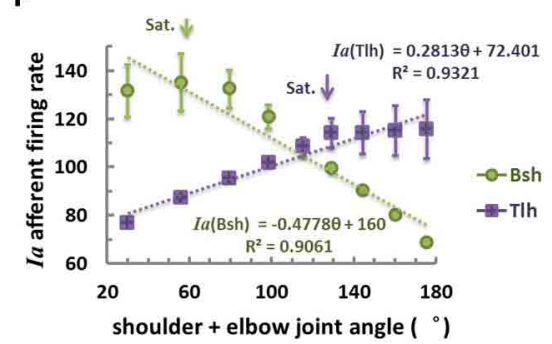

FIGURE 7 | The linear fusimotor control strategy, $\theta_{\mathrm{EP}}-\gamma_{\mathrm{S}}$ curves (A-C), and $l a$ afferents response, $\left(\theta_{E P}-l a\right)$ curves of muscle spindles (D-F), for all muscles. The $(\mathbf{A}, \mathbf{D})$ is for shoulder actuators, the $(\mathbf{B}, \mathbf{E})$ is for elbow actuators, and the $\mathbf{( C , F )}$ is for bi-articular muscles. The axes were defined the same as in Figure 6. When $\gamma_{\mathrm{s}}$ inputs of muscle spindles are linearly modulated with joint angles from 0.3 to 0.9 , the firing rates of $l a$ afferents do not display a linear and monotonical relation to joint angles. Saturations of la responses occur at each muscle, as indicated by the arrows. This is due to the nonlinear physiological properties of muscle spindle demonstrated in Figure 5, and would not be possible to avoid if the full range of fusimotor commands were to be used to encode joint angle information.

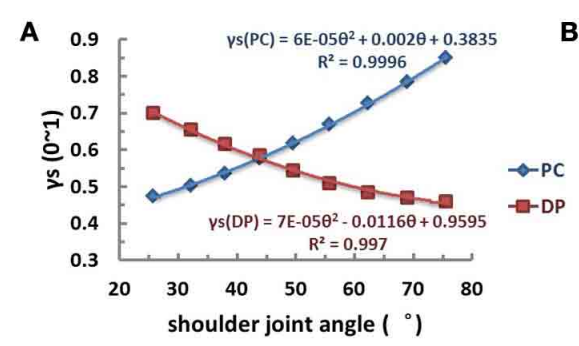

D

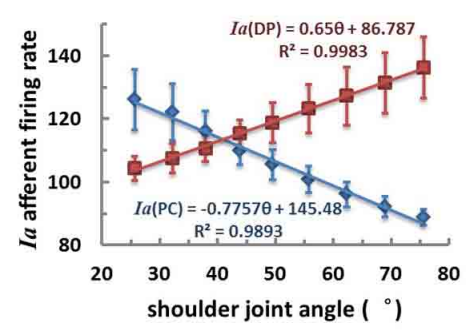

E

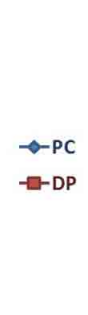

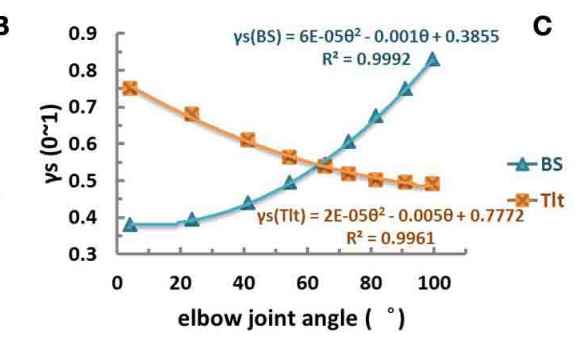

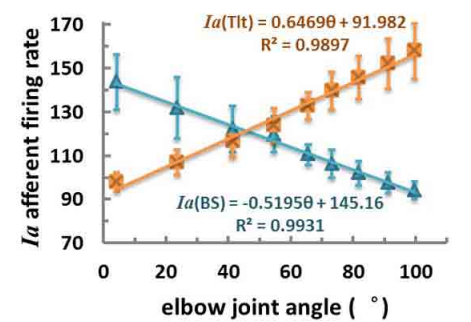

$\mathbf{F}$
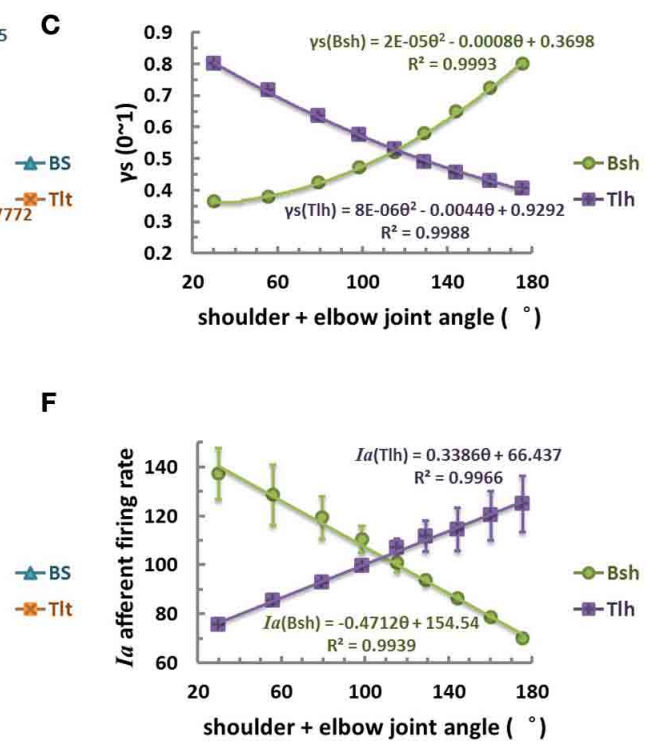

FIGURE 8 | The quadratic strategy of fusimotor control, $\theta_{\mathrm{EP}}-\gamma_{\mathrm{s}}$ curves $(A-C)$, and $l a$ afferents response, $\left(\theta_{E P}-l a\right)$ curves of muscle spindles (D-F), for each muscle. The (A,D) is for shoulder actuators, the $\mathbf{B}, \mathbf{E})$ is for elbow actuators, and the $\mathbf{( C , F )}$ is for bi-articular muscles. The axes were defined the same as in Figure 6. When $\gamma_{\mathrm{s}}$ inputs of muscle spindles are modulated with joint angles $\theta_{E P}$ quadratically in a monotonical manner with joint angles from 0.3 to 0.9 , the firing rates of la afferents show a robust linear relation with joint angles with an average goodness of linear fitting $R^{2}>0.99$. This phenomenon suggests that it is possible to manipulate the fusimotor commands to linearize the nonlinear la sensitivity revealed in Figure 5. hypothetical fusimotor control strategy must reproduce all experimentally observed behaviors simultaneously. Strategies that do not simultaneously satisfy these necessary conditions could not be considered biologically plausible.
With this criterion in mind, we used a computational approach to address these related issues, (1) what specific kinematic information is encoded in gamma static fusimotor efferents? And (2) how gamma static command may be controlled in order 
Table 3 | Fitting coefficients of quadratic $\gamma_{\mathrm{s}}$ strategy.

\begin{tabular}{|c|c|c|c|c|c|c|c|}
\hline & \multicolumn{3}{|c|}{$\gamma_{s}=a \theta^{2}+b \theta+c$} & \multicolumn{4}{|c|}{$\mathrm{la}=\mathrm{k} \theta+\mathrm{e}$} \\
\hline PC & $6 e-5$ & 0.0020 & 0.3835 & 0.9996 & -0.7757 & 145.48 & 0.9893 \\
\hline DP & $7 e-5$ & -0.0116 & 0.9595 & 0.9970 & 0.6500 & 86.787 & 0.9983 \\
\hline BS & $6 e-5$ & -0.0010 & 0.3855 & 0.9992 & -0.5195 & 145.16 & 0.9931 \\
\hline Tlt & $2 e-5$ & -0.0050 & 0.7772 & 0.9961 & 0.6469 & 91.982 & 0.9897 \\
\hline Average & & - & & 0.9983 & 0.5 & & 0.9935 \\
\hline
\end{tabular}

to maintain a consistent $I a$ encoding of joint angles during movements. The peripheral factors that may affect the outcome of central coding were examined first with the VA model in Figures 3-5. It was interesting to note that the variability caused by the internal SDN noise simplified the relation between muscle fascicle length and joint angles in certain degree to a proximately linear relation within the operating range of joint angles, because of the averaging effects as shown in Figure 3. This appeared to lessen the nonlinear effects in the peripheral musculoskeletal system. However, the spindle sensitivity did show a significant nonlinear response that could affect both central and peripheral coding of joint angles (Figure 5). With prominent nonlinear spindle response, three hypotheses were tested regarding the central coding strategies of joint angles. The first set of simulation rejected the hypothesis that a constant $\gamma_{\mathrm{s}}$ control may be a plausible neural strategy in spite of its excellent linear $\theta_{\mathrm{EP}}-\mathrm{Ia}$ relation. Experimental evidence clearly showed that a dynamic pattern of $\gamma_{s}$ modulation was observed to covary with $\alpha$ command and joint angles during movements (Taylor et al., 2000, 2004, 2006). In the test of second hypothesis of this study, the linear $\theta_{\mathrm{EP}}-\gamma_{\mathrm{s}}$ modulation did not produce a well-regulated linear $\theta_{\mathrm{EP}}-\mathrm{Ia}$ relation, which was observed in experimental recordings in man and animals (Cordo et al., 2002; Stein et al., 2004). This outcome may be attributable to the nonlinear sensitivity presented in the spindle response in Figure 5. Thus, the hypothesis of linear control strategy of spindle sensitivity expressed by linear $\theta_{\mathrm{EP}}-\gamma_{\mathrm{s}}$ correlation was also rejected. Then, we tested the third hypothesis, in which a nonlinear control strategy of $\gamma_{s}$ commands may avoid the nonlinear zone in the landscape of spindle sensitivity in Figure 5. The results indicated that a second order nonlinear relation between $\gamma_{s}$ command and joint angle, i.e., a parabolic $\theta_{\mathrm{EP}}-\gamma_{\mathrm{s}}$ curve, was indeed necessary, in order for the I $a$ afferent to be linearly correlated with joint angle. With the quadratic strategy, the Ia afferents of both mono-articular and bi-articular muscles displayed the similar property of a robust linear relation with joint angles. This result implies that the brain could learn the peripheral constraints, and program the nonlinear central coding of the monotonic $\theta_{\mathrm{EP}}-\gamma_{\mathrm{s}}$ curve, and send the $\gamma_{\mathrm{s}}$ command to inform the periphery about the centrally planned joint angles. Such central coding strategy would also allow the spindle to maintain an accurate and consistent encoding of angular information in
Ia afferents, which the brain needs to evaluate the peripheral performance.

Lastly, we calculated the average position sensitivity of $I a$ afferents of all six muscles from simulation results for the three strategies of fusimotor control. The slopes of the linear $\theta_{\mathrm{EP}}-I a$ relationship were compared to that of Cordo's et al. (2002) human physiological recordings. The average position sensitivity under constant gamma control was $1.04 \pm 0.29 \mathrm{pps} /{ }^{\circ}$ (Table 2), which was much higher than that of quadratic strategy of gamma modulation of $0.57 \pm 0.16 \mathrm{pps} /{ }^{\circ}$ (Table 3). The latter was closer to that of position sensitivity of holding rate measured by Cordo et al. (2002), which was $0.40 \pm 0.30 \mathrm{pps} /{ }^{\circ}$. This further supports the quadratic hypothesis as a plausible fusimotor control strategy for postures and movements.

The implication that fusimotor signals encode kimematic information of planned (or desired) movements is consistent with the finding that fusimotor activities were enhanced when performing a naïve task (Hulliger, 1984). When a new movement is performed, the CNS needs to program an optimal pattern of kinematics that is represented in fusimotor commands. This may necessitates modifying the planned kinematics frequently from practice to practice. The heightened activities seen in the fusimotor signals suggest that a process of searching for optimal kinematics is going on for the new task. In this process, proprioceptive afferents are used to assess the outcome of motor action, and are compared to the centrally programmed kinematics to detect any deviations between the programmed movement and outcome movement. Modifications are made in both centrally planned kinematics (gamma commands) and motor actions (alpha commands) to further optimize movement performance. Thus, the motor learning and control system acts like a reference adaptive control system, where the gamma commands provide the reference trajectories of movement (Taylor et al., 2006), and the alpha commands produce optimal driving inputs to the extrafusal muscle fibers. To fully appreciate the reference adaptive control of movement, central encoding of dynamic fusimotor commands with respect to movement kinematics should also be elucidated in the future research.

\section{CONCLUSION}

We examined the peripheral factors that may influence the central and peripheral coding of joint angles through efferent and 
afferent innervations of the spindle apparatus. Based on these peripheral constraints, we have tested three hypotheses regarding static fusimotor control strategies of mono-articular and biarticular muscles to achieve a reliable encoding and decoding of joint angle information. Results suggest that a quadratic strategy of static fusimotor control could lead to a linear relation between I $a$ afferents and joint angle with an average sensitivity close to the experimental value. This suggests that the $\gamma_{s}$ command encodes joint position information in the CNS with a parabolic $\theta_{\mathrm{EP}}-\gamma_{\mathrm{s}}$ curve. Under the strategy of quadratic $\gamma_{s}$ control, the peripheral

\section{REFERENCES}

Boyd, I. A. (1980). The isolated mammalian muscle spindle. TINS 258-265.

Boyd, I. A., Murphy, P. R., and Moss, V. A. (1985). "Analysis of primary and secondary afferent responses to stretch during activation of the dynamic bag1 fiber or the static bag2 fiber in cat muscle spindle," in The Muscle Spindle, eds I. A. Boyd and M. H. Gladden (London: Macmillan), 153-158

Boyd, I. A., and Smith, R. S. (1984). "The muscle spindle," in Peripheral Neuropathy, 2nd Edn, eds R. J. Dyck, P. K. Thomas, E. H. Lombert, and R. Bunge (Philadelphia, PA: W.B. Saunders Company), 171-202.

Cheng, E. J., Brown, I. E., and Loeb, G. E. (2000). Virtual muscle: a computational approach to understanding the effects of muscle properties on motor control. J. Neurosci. Methods $101,117-130$

Corcos, D. M., Gottlieb, G. L., and Agarwal, G. C. (1989). Organizing principles for single joint movements, II. A speedsensitive strategy. J. Neurophysiol. 62, 358-368.

Cordo, P. J., Flores-Vieira, C., Verschueren, S. M. P., Inglis, J. T., and Gurfinkel, V. (2002). Position sensitivity of human muscle spindles: single afferent and population representations. $J$. Neurophysiol. 87, 1186-1195.

Feldman, A. G. (1986). Once more on the equilibrium-point hypothesis $(\lambda$ model) for motor control. J. Motor Behav. 18, 17-54.

Flash, T., and Hogan, N. (1985). The coordination of arm movements: an experimentally confirmed mathematical model. J. Neurosci. 5, 1688-1703.

Ghez, C., Scheidt, R. A., and Heijink, H. (2007). Different learned coordinate frames for planning trajectories and final positions in reaching. $J$. Neurophysiol. 98, 3614-3626.

Gottlieb, G. L., Corcos, D. M., and Agarwal, G. C. (1989). Organizing principles for single joint movements, I. A speed-insensitivity strategy. J. Neurophysiol. 62, 342-357.

Granit, R. (1970). The Basis of Motor Control. London: Academic Press.

Harris, C. M., and Wolpert, D. M. (1998). Signal-dependent noise determines motor planning. Nature $394,780-784$

Hasan, Z. (1986). Optimized movement trajectories and joint stiffness in unperturbed inertially loaded movements. Biol. Cybern. 53, 373-382.

He, X., Du, Y. F., and Lan, N., (2012). "Validation of a virtual arm model for movement control and rehabilitation," in Proceedings of IEEE/EMBS International Conference on Biomedical and Health Informatics (BHI 2012), (Shenzhen, China)

He, X., and Lan, N. (2011). "Central and peripheral coding of joint position by descending gammastatic commands and muscle spindle afferents," in Proceedings of 5th International IEEE EMBS Conference on Neural Engineering, (Cancun, Mexico), April 2011.

Houk, J. C., and Rymer, Z. (1981). "Neural control of muscle length and tension," in Handbook of Physiology-The Nervous System, Vol. II. Motor Control, Part, I, eds J. M. Brookhart, V. B. Mountcastle and V. B. Brooks (Bethesda, MD: American Physiological Society), 189-228.

Hulliger, M. (1984). The mammalian muscle spindle and its central control. Rev. Physiol. Biochem. Pharmacol. 101, 1-110.

Hulliger, M., Horber, F., Medved, A., and Prochazka, A. (1987). An experimental simulation method for iterative and interactive reconstruction of unknown (fusimotor) inputs contributing to known (spindle afferent) responses, $J$. Neurosci. Methods 21, 225-238.

Hulliger, M., and Prochazka, A. (1983). A new stimulation method to deduce fusimotor activity from

linear $\theta_{\mathrm{EP}}-$ Ia relation could be maintained, and used to decode actual angular information reliably from Ia afferents.

\section{ACKNOWLEDGMENTS}

Materials of this paper are based on the work supported by a 973 basic research grant from the Ministry of Science and Technology of China (No. 2011CB013304), a grant from the Natural Science Foundation of China (No. 31070749) and a doctoral training grant from the Ministry of Education of China (No. 20100073110064).

afferent discharge recorded in freely moving cats. J. Neurosci. Methods 8 197-204.

Jones, K. E., Hamilton, A. F. C., and Wolpert, D. M. (2002). Sources of signal-dependent noise during isometric force production. J. Neurophysiol. 88, 1533-1544.

Kawato, M., Maeda, Y., Uno, Y., and Suzuki, R. (1990). Trajectory formation of arm movement by cascade neural network model based on minimum torque-change criterion. Biol. Cybern. 62, 275-288.

Kurtzer, I., Herter, T. M., and Scott, S. H. (2005). Random change in cortical load representation suggests distinct control of posture and movement. Nat. Neurosci. 8 , 498-505.

Lan, N. (1997) Analysis of an optimal control model of multi-join arm movements. Biol. Cybern. 76, 107-117.

Lan, N., and Crago, P. C. (1994) Optimal control of antagonist muscle stiffness during voluntary movements. Biol. Cybern. 70, 397-405.

Lan, N., Li, Y., Sun, Y., and Yang, F. S. (2005). Reflex regulation of antagonist muscles for control of joint equilibrium position. IEEE Trans. NSRE 13, 60-71.

Lemon, R. N. (2008). Descending pathways in motor control. Annu. Rev. Neurosci. 31, 195-218.

Matthews, P. B. C. (1962). The differentiation of two types of fusimotor fibre by their effects on the dynamic response of muscle spindle primary endings. Q. J. Exp. Physiol. 47, 324-333.

Matthews, P. B. C. (1981). "Muscle spindles: their messages and their fusimotor supply," in Handbook of Physiology-The Nervous System, Vol. II. Motor Control, Part, I, eds J. M. Brookhart, V. B. Mountcastle, and V. B. Brooks (Bethesda, MD American Physiological Society), 189-228.

Merton, P. A. (1953). "Speculations on the servo-control of movmenet," in Ciba Foundation Symposium, the Spinal Cord, ed G. E. W.
Wolstenholme (London: Churchill Press), 247-255.

Mileusnic, M. P., Brown, I. E., Lan, N., and Loeb, G. E. (2006) Mathematical models of proprioceptors. I. Control and transduction in the muscle spindle. J. Neurophysiol. 96, 1772-1788.

Scheidt, R. A., and Ghez, C. (2007). Separate adaptive mechanisms for controlling trajectory and final position in reaching. J. Neurophysiol. 98, 3600-3613.

Song, D., Lan, N., Loeb, G. E., and Gordon, J. (2008a). Model-based sensorimotor integration for multijoint control, development of a virtual arm model. Ann. Biomed. Eng. 36, 1033-1048.

Song, D., Raphael, G., Lan, N., and Loeb, G. E. (2008b). Computationally efficient models of neuromuscular recruitment and mechanics. J. Neural Eng. 5, 175-184.

Stein, R. B. (1974). Peripheral control of movement. Physiol. Rev. 54, 215-243.

Stein, R. B., Weber, D. J., Aoyagi, Y., Prochazka, A., Wagenaar, J. B. M., Shoham, S., and Normann, R. A. (2004). Coding of position by simultaneously recorded sensory neurons in the cat dorsal root ganglion. J. Physiol. 560, 883-896.

Taylor, A., and Donga, R. (1989). "Central mechanisms of selective fusimotor control," in Afferent Control of Posture and Locomotion, Progress in Brain Research, Vol. 80, eds J. H. J. Allum and M. Hulliger, 27-35.

Taylor, A., Durbaba, R., and Ellaway, P. H. (2004). Direct and indirect assessment of gamma motor firing patterns. Can. J. Physiol. Pharmacol. 82, 793-802.

Taylor, A., Durbaba, R., Ellaway, P. H., and Rawlinson, S. (2006). Static and dynamic $\gamma$-motor output to ankle flexor muscles during locomotion in the de-cerebrate cat. J. Physiol. 571, 711-723.

Taylor, A., Ellaway, P. H., Durbaba, R., and Rawlinson, S. (2000). 
Distinctive patterns of static and dynamic gamma motor activity during locomotion in the de-cerebrate cat. J. Physiol. 529, 825-836.

Todorov, E., and Jordan, M. I. (2002). Optimal feedback control as a theory of motor coordination. Nature 5, 1226-1235.

Uno, Y., Kawato, M., and Suzuki, R. (1989). Formation and control of optimal trajectory in human multijoint arm movement: minimum torque-change model. Biol. Cybern. 61, 89-101.

Vallbo, A. B., and al-Falahe, N. A. (1990). Human muscle spindle response in a motor learning task. $J$. Physiol. 421, 553-568.

Wand, P., and Schwarz, M. (1985). Two types of cat static fusimotor neurons under separate central control? Neurosci. Lett. 58, 145-149.
Conflict of Interest Statement: The authors declare that the research was conducted in the absence of any commercial or financial relationships that could be construed as a potential conflict of interest.

Received: 10 April 2012; accepted: 13 August 2012; published online: 30 August 2012.

Citation: Lan $N$ and $\mathrm{He} X$ (2012) Fusimotor control of spindle sensitivity regulates central and peripheral coding of joint angles. Front. Comput. Neurosci. 6:66. doi: 10.3389/fncom.2012.00066 Copyright (C) 2012 Lan and He. This is an open-access article distributed under the terms of the Creative Commons Attribution License, which permits use, distribution and reproduction in other forums, provided the original authors and source are credited and subject to any copyright notices concerning any thirdparty graphics etc. 


\section{APPENDIX}

LIST OF ACRONYMS AND SYMBOLS

$\alpha$-Alpha motoneurons

$\alpha_{d}, \alpha_{\text {dyn }}$-Alpha dynamic command

$\alpha_{s}, \alpha_{\text {stat }}$-Alpha static command

BS-Brachialis

Bsh-Biceps Brachii short head

$\gamma$-Gamma motoneurons

$\gamma_{\mathrm{d}}, \gamma_{\mathrm{dyn}}$-Gamma dynamic command

$\gamma_{s}, \gamma_{\text {stat }}$-Gamma static command

CNS-Central Nervous System

DOF-Degree of freedom

DP-Deltoid Posterior

$\mathrm{ECRb}$-Extensor Carpi Radialis brevis

ED-Extensor Digitorum

EMG-Electromyography

$\theta_{\mathrm{EP}}-$ Equilibrium point angle

$F_{\mathrm{m}}$ - Muscle force

GABA- $\gamma$-aminobutyric acid

GTO-Golgi Tendon Organ

Ia - Primary afferent from spindle

$\mathrm{I} b$-afferent from GTO

II-Secondary afferent from spindle

$L_{\mathrm{ce}}$-Muscle fascicle length

$L_{\mathrm{mt}}-$ Musculo-tendon length

PC-Pectoralis major Clavicle portion

pps-pulse per second

ROM-Range of Motion

SDN_Signal Dependent Noise

SNR—Substantia Nigra

Tlh-Triceps Brachii long head

Tlt-Triceps Brachii lateral head

VA-Virtual Arm

VM-Virtual Muscle 